\title{
Prenatal Diagnosis of Morbidly Adherent Placenta with 2D Ultrasonography, 3D Color Power Doppler and Magnetic Resonance Imaging
}

\author{
${ }^{1}$ Sawsan Al Obaidly, ${ }^{2}$ Asim Kurjak \\ ${ }^{1}$ Department of Obstetrics and Gynecology, Women's Hospital, Hamad Medical Corporation, Doha, Qatar \\ ${ }^{2}$ Department of Obstetrics and Gynecology, Medical School University of Zagreb, Croatia
}

Correspondence: Sawsan Al Obaidly, Department of Obstetrics and Gynecology, Women's Hospital, Hamad Medical Corporation Doha, Qatar, e-mail: iris.qa@gmail.com

\begin{abstract}
The incidence of placenta accreta/percreta should rise steadily over the next century as the frequency of cesarean sections and advanced maternal age, both independent risk factors, increases. Patients who are at risk should be identified. The diagnosis of placenta previa accreta/percreta is possible by using gray-scale sonography, conventional color Doppler imaging and MRI through studying the relation of placenta to the uterine wall and nearby pelvic structures.

The potentially new modality of 3D and 3D color power Doppler ultrasound has it's value as a tool to achieve significantly increased diagnostic accuracy in the prediction of massive hemorrhage by assessing the extent, location and quantification of abnormal uteroplacental neovascularization. Hence, 3D ultrasound has the potential for providing additional information over conventional 2D ultrasound studies in the diagnosis of placenta previa percreta.

The diagnosis and anticipation of the problem achieve the best results for the obstetrician and the patient.

Keywords: Placenta accrete/percreta, 2D ultrasonography, 3D color power Doppler, MRI.
\end{abstract}

\section{INTRODUCTION}

For the last century an increase rate of cesarean section was noted hence the mortality and morbidity of repeated cesarean section, mainly placenta previa and morbidly adherent placenta.

The introduction of obstetrics ultrasound with Doppler and the latest development of 3D and 3D color and power Doppler in modern obstetrics helped in the early detection and management of adherent placenta. With such achievement these low cost modalities might replace the MRI completely for the diagnosis of morbidly adherent placenta.

\section{CASE REPORT}

We present a case of a 34 years old lady. Her previous 3 babies have been delivered at term, previous two with LSCS.

She had a scan at around 22 weeks showing single viable breech with normal fetal anatomy but, anterior placenta previa type 4 with no evidence of invasion. Cervical length was $2.1 \mathrm{~cm}$. MRI was done around the same period which confirmed central placenta previa without invasion.

Patient was seen regularly in the clinic and she was always asymptomatic, never had any episode of vaginal bleeding or abdominal pain or hematuria and she had a normal perception of fetal movement.

Fetal growth scan was normal at 28 weeks which again showed placenta previa type 4 but, this time with retroplacental neovascularization suggesting placenta increta/percreta.

The MRI at 31 weeks gestation confirmed the ultrasound result of increta/acreta central anterior placenta previa (Figs $1 \mathrm{~A}$ and $\mathrm{B})$.

She was scanned at 34 weeks using 2D gray scale and color Doppler (Figs 2 and 3), then 3D color, power Doppler (Figs 5 to 8) which showed presence of abnormal placental lacunae, irregularity and loss of retroplacental sonoluscent area, thinning or disruption of the hyperechoic uterine serosa-bladder interface, and presence of exophytic masses invading the urinary bladder.

Color Doppler criteria are illustrated on the Figures 4A to 5: diffuse or focal lacunae flow pattern, sonoluscent vascular lakes with turbulent flow typified by high velocity and low resistance waveform, hypervascularity of the uterine-bladder interface with abnormal blood vessels linking placenta and bladder, and markedly dilated blood vessels over the peripheral subplacental region. 



Figs 1A and B: MRI on T2-weighted images, sagittal and PA coronal views of pelvis showing anterior placenta previa with interrupted uterine wall and uterine bulging with tenting of the bladder wall posteriorly through direct placental invasion

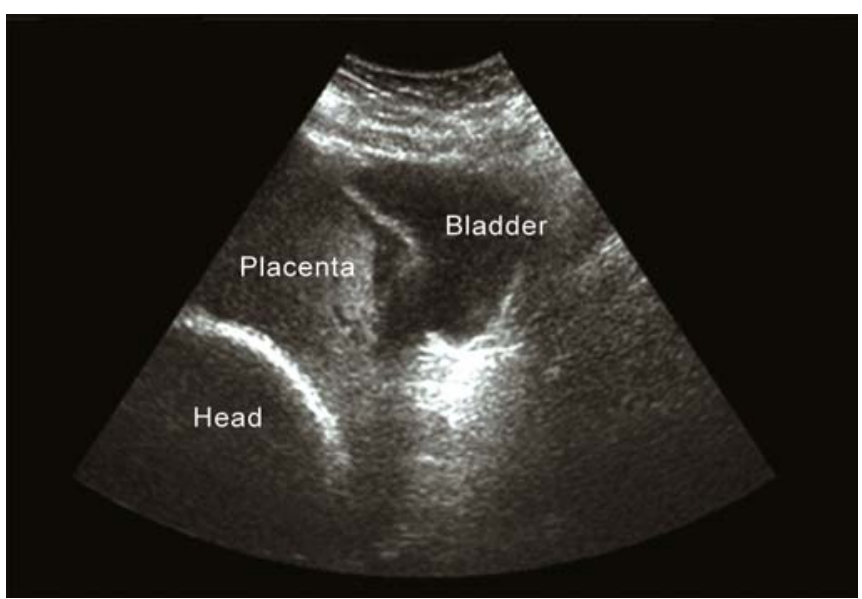

Fig. 2: 2D gray scale ultrasound picture at 34 weeks gestation showing anterior placenta previa major, loss of normal hypoechoic retroplacental myometrial zone with focal disruption of uterine serosa and interruption of posterior bladder wall uterine interface with appearance of bladder exophyte

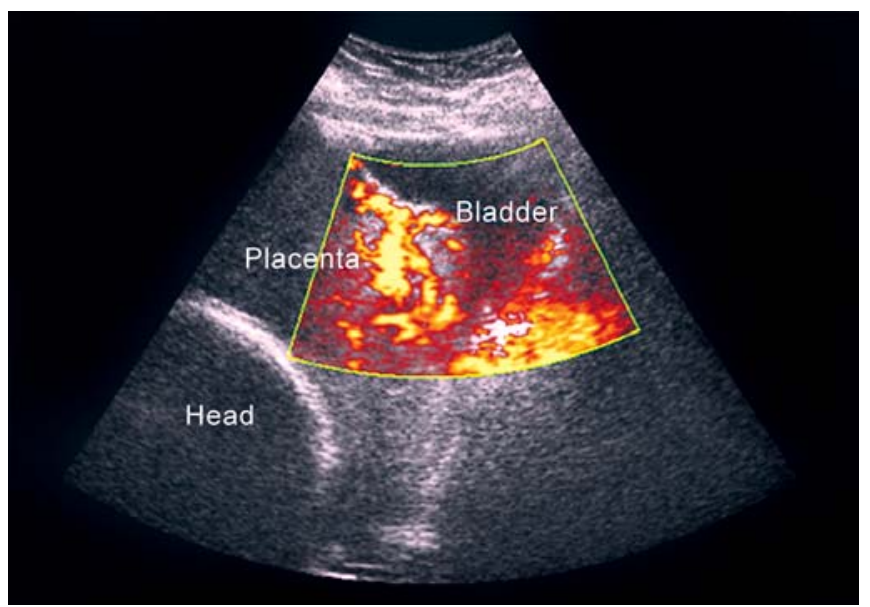

Fig. 3: 2D power Doppler ultrasound picture at 34 weeks gestation showing the abnormal vascularity between placenta and the bladder
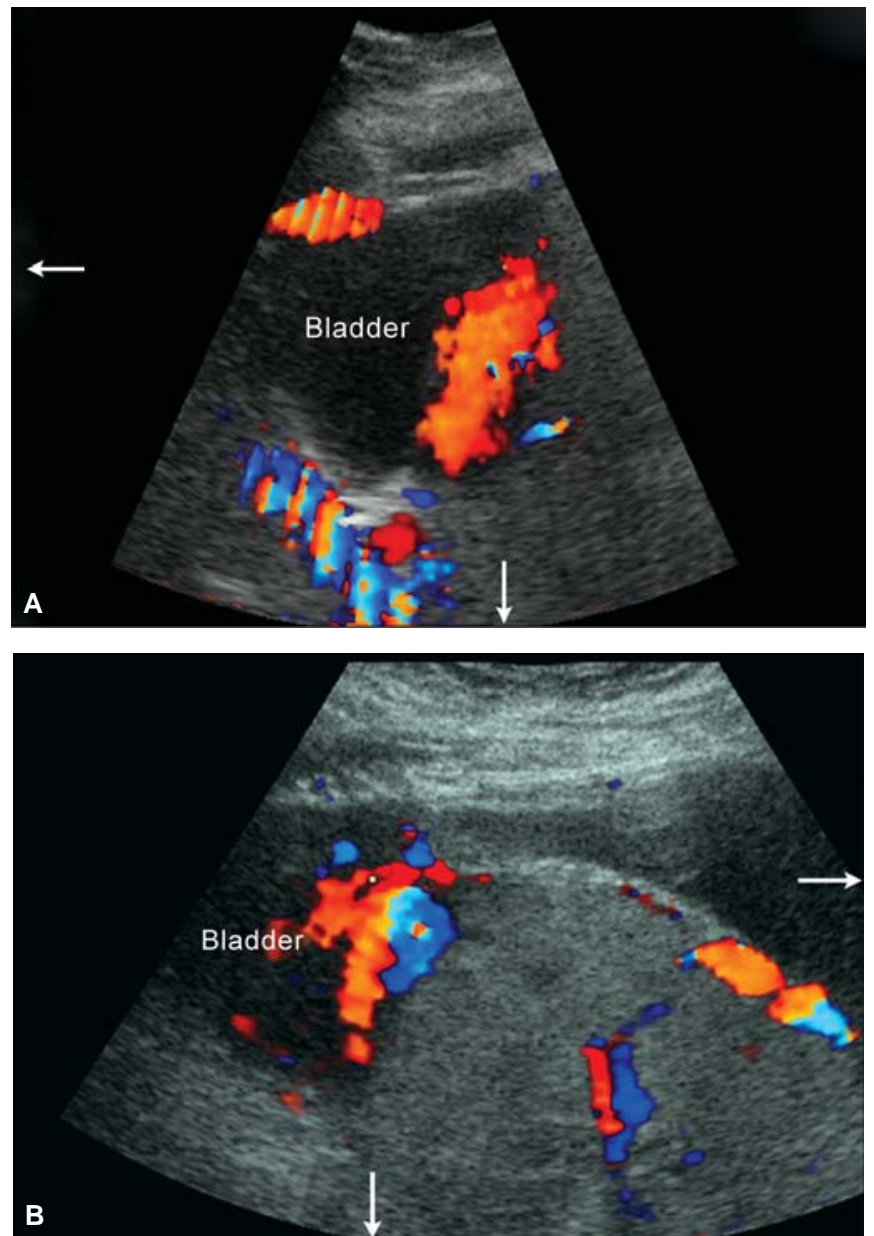

Figs 4A and B: 3D color Doppler. Lateral and basal view of the bladder showing abnormal large vessels invading through bladder wall posteriorly 


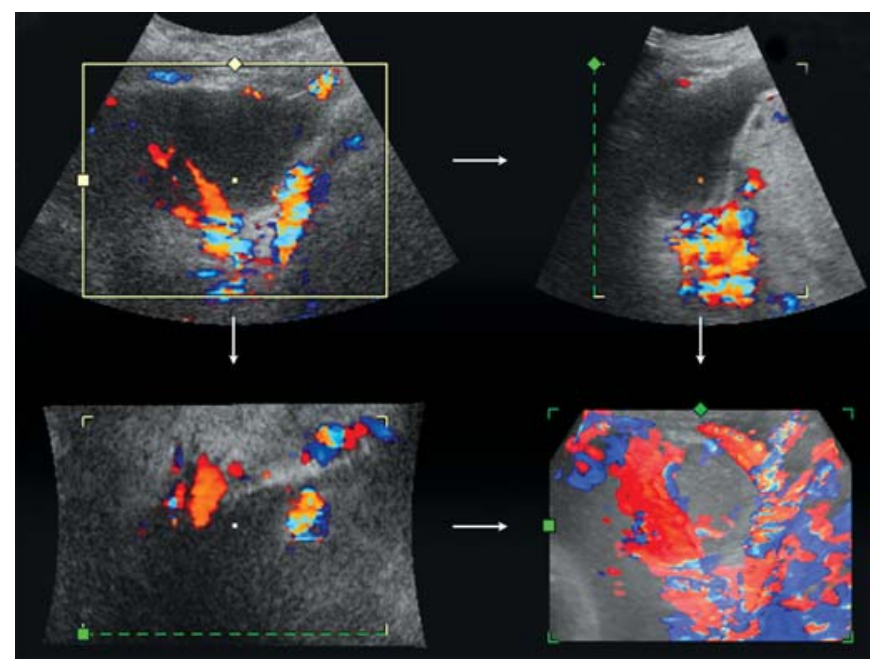

Fig. 5: 3D color Doppler showing abnormal branching vascular tree of placenta and around the bladder. There is hypervascularity of the uterine-bladder interface with abnormal blood vessels linking placenta and bladder, markedly dilated blood vessels over the peripheral sub placental region

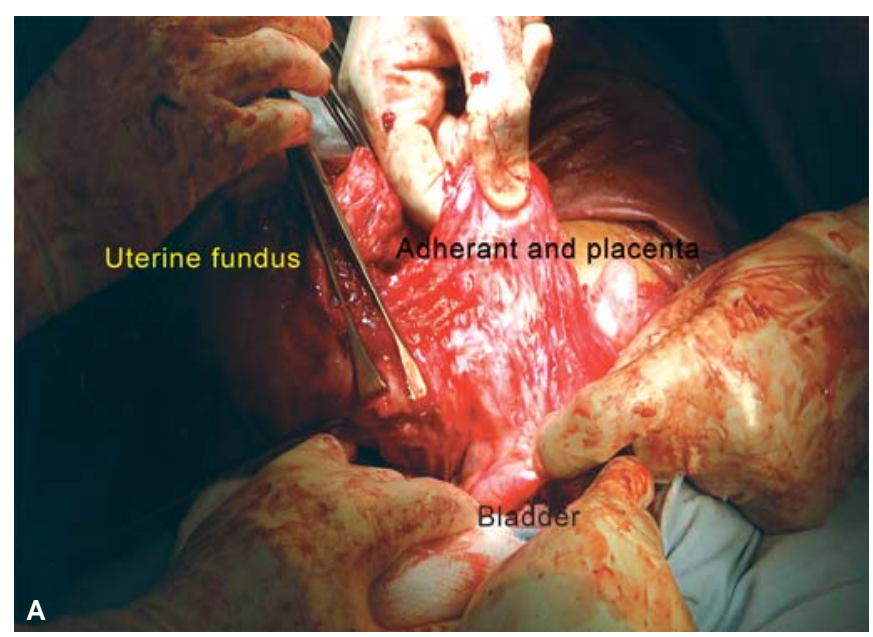

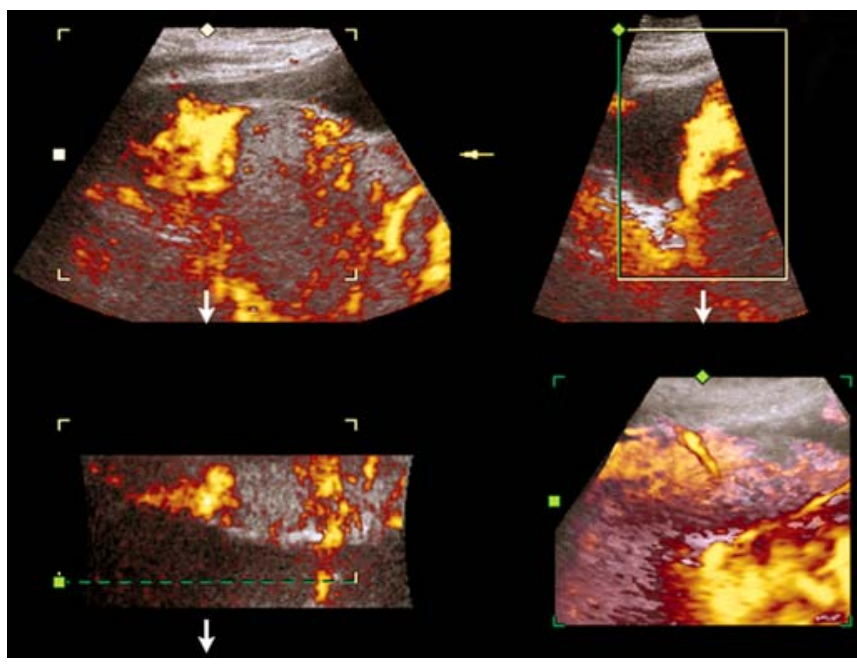

Fig. 6: 3D power Doppler—Lateral view: Intraplacental high vascularity, inseparable cotyledonal and intervillous circulation, tortuous vascularity with chaotic branching. Basal view: Numerous coherent vessels involving serosa bladder interface

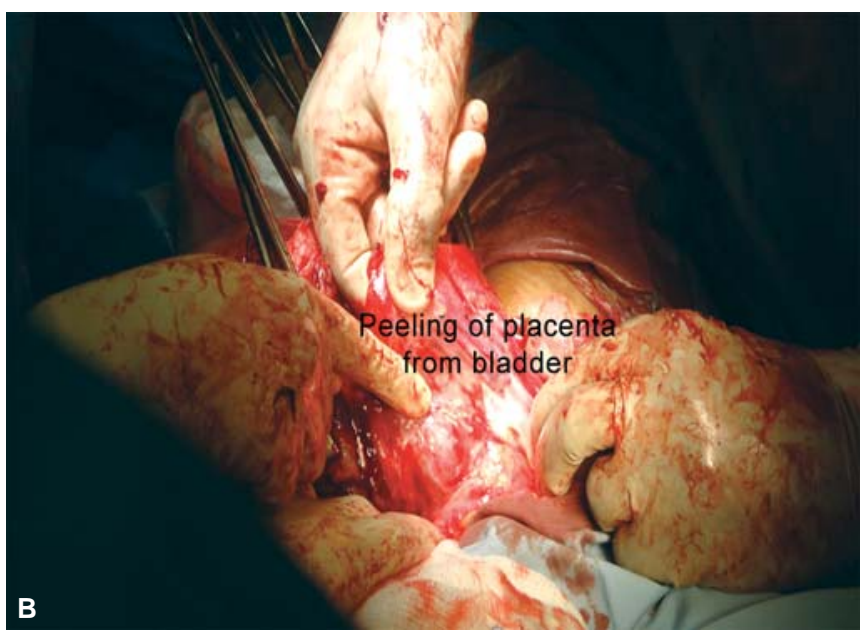

Figs $7 \mathrm{~A}$ and B: Intraoperative finding of placenta percreta attached to the bladder wall posteriorly

\section{D Power Doppler Criteria (Fig. 6)}

Lateral view: Intraplacental high vascularity, inseparable cotyledonal and intervillous circulation, tortuous vascularity with chaotic branching.

Basal view: Numerous coherent vessels involving serosa bladder interface.

The patient had an elective hospital admission at 34 weeks. She was posted for a cesarean hysterectomy at 36 weeks. Blood was prepared; senior anesthetist was involved; urologist was consulted and planed to attend the delivery; vascular surgeon was also aware of the case and could have been available in the theatre when needed. The night preceding the surgery the patient complained of painless vaginal bleeding but she was hemodynamically stable and the bleeding stopped so awaited the morning time when she had bilateral uterine artery catheters inserted by an experienced interventional radiologist.

During surgery phanenstill skin incision was done, then a high transverse uterine incision was done to deliver a live baby girl who weighed $3 \mathrm{~kg}$ with good Apgar score. The placenta was central, anterior covering the internal os and placental tissue was invading myometrium reaching outside to bladder serosa (Fig. 7A).

The placenta was cut during the uterine incision and it was bleeding, attempt to deliver the placenta which was removed partially. Subtotal hysterectomy was undertaken with pealing of the placenta from the anteroposterior bladder wall serosa (Figs 7B and 8). 


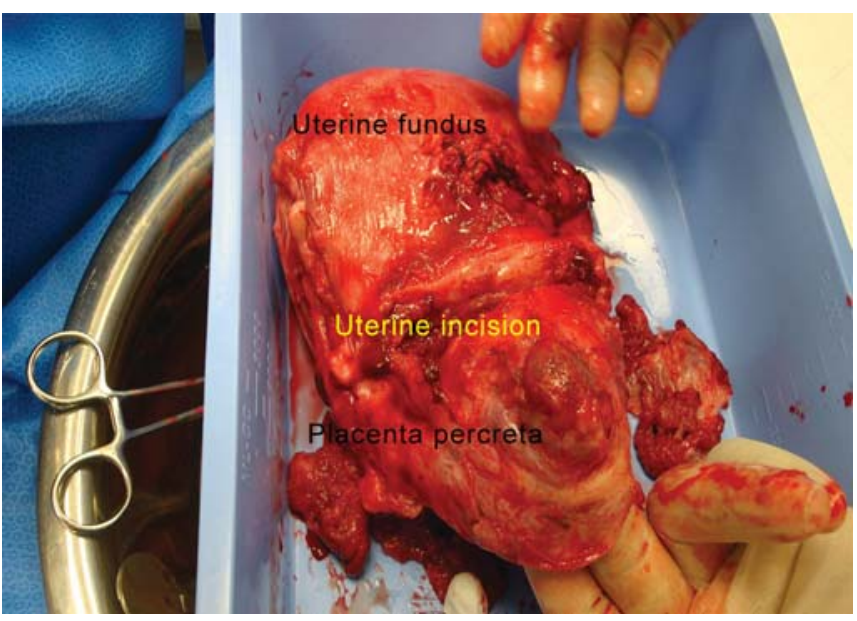

Fig. 8: Specimen of the uterus showing placenta percreta anteriorly

Urologists were available and clear identification of the ureters done. The bladder wall seemed to be intact. The patient had lost 6 liters of blood and good blood replacement was given. Patient was transferred to the surgical ICU and she was transferred to the High dependency unit a day later. The uterine artery catheters were not used and were removed after. Patient remained hospitalized for eight days and discharged home with her baby, both in good condition.

\section{DISCUSSION}

Implantation of the placenta across the internal cervical os constitutes placenta previa. The condition increases maternal and fetal morbidity and also the need for emergency cesarean delivery, owing to profuse bleeding in undiagnosed cases. ${ }^{1,2}$ The incidence of placenta previa is estimated to be 0.14 to $0.5 \%$ at term. ${ }^{1-3}$ A placenta that is attached directly to the uterine muscle without intervening decidua is termed placenta accreta, whereas placenta increta and percreta refer to partial and complete penetration of such a placenta through the uterine wall. Placenta accreta occurs in approximately $10 \%$ of cases of placenta previa ${ }^{4,5}$ whereas placenta increta and percreta are seen even less frequently. ${ }^{5}$

Placenta percreta is the most serious of placental implantation anomalies beyond the uterine serosa. It can unexpectedly lead to catastrophic blood loss, multiple complications such as adult respiratory distress syndrome, Sheehan's syndrome, renal failure, and even death. Maternal mortality and morbidity are significantly increased by placenta percreta. The mortality can be as high as $10 \%{ }^{6}$ The morbidity included bladder laceration (20\%), urinary fistula (13\%), ureteral transaction (6\%), and a resulting small bladder capacity (4\%). Partial cystectomy was performed in $44 \%$ of cases. ${ }^{7}$
The diagnosis of placenta accreta can be difficult but is possible using gray scale ultrasound and color Doppler imaging. ${ }^{8,9}$ Placental penetration through the uterine wall has been recently described by investigators using ultrasound and magnetic resonance imaging (MRI). ${ }^{9}$ There is a need for reliable antenatal diagnosis for these serious conditions. If these pregnancies can be identified before delivery, the site and time of delivery as well as the surgical approach can be planned ahead and decrease the blood loss. ${ }^{10}$

The medical and surgical approach for our patient was fairly appropriate. The catastrophic event was properly anticipated. The 2D ultrasound did not exclude placenta increta, percreta at 22 weeks. However, as the radiologist commented on the short cervix, this could have been early changes in the placental localization beyond the decidual lining. While the repeated 2D ultrasound at 28 weeks fulfilled the diagnostic features of placenta accreta, including loss of normal hypoechoic retroplacental myometrial zone, focal disruption of the uterine serosa and surrounding tissues, and intraplacental lacunae were found. These grayscale ultrasonographic findings appear to be typical of placenta accreta as described by Taipale et al. ${ }^{2}$ Furthermore, color Doppler imaging revealed increased vascularity between the placenta and myometrium, which has not always been documented in cases of placenta accreta. ${ }^{9}$

Chou et $\mathrm{al}^{11}$ reports that seven of the 45 patients exhibited characteristic 2D US findings for placenta increta/ percreta. The 2D US criteria for placenta increta/percreta included placental lacunae, absence of the retroplacental clear zone, and interruption of posterior bladder wall-uterine interface. Among these seven patients of advanced invasive placentation, a targeted scan with adjunctive threedimensional (3D) US correctly provided additional convincing information to the indeterminate diagnosis by 2D US in these patients who were affected with variable degrees of bladder wall involvement found at surgery.

\section{MR IMAGING}

Thinning of the uterine wall and loss of the hypoechoic area between the placenta and myometrium were found by MRI in the case of placenta percreta. Baughman et $\mathrm{al}^{12}$ described MRI characteristics of placenta accreta: Placenta previa, uterine bulging, heterogeneous signal intensity within the placenta, dark intraplacental bands on T2-weighted images, focal interruptions in the myometrial wall, tenting of the bladder, direct visualization of the invasion of pelvic structures by placental tissue.

Antenatal suspicion of placenta percreta made possible the appropriate preoperative and perioperative management, which has also been described by Tourne et al. ${ }^{13}$ In our 
case, uterine artery catheters were inserted preoperatively as prophylactic measure but embolization was not performed while other cases as Taipale et al performed embolization of the uterine arteries after cesarean delivery and before hysterectomy to minimize bleeding, which is recommended in the management of severe postpartum bleeding. ${ }^{13}$

The repeated ultrasound for our patient at 34 weeks with the aid of 3D ultrasound showed more details about placental invasion. The tentative proposed 3D US criteria for advanced invasive placentation of suspected bladder involvement are as follows: (1) a loss of the echolucent space between the bladder and the placenta in coronal and axial scans; (2) invasion of the bladder by the infiltrating placenta with irregularity and disruption of the normal bladder wall architecture and/or a focal exophytic placental mass projecting into the bladder in coronal and axial scans; and (3) aberrant blood vessels in the spatial vascular network in the region of interest extending into the bladder by rotational angiography. ${ }^{11,14}$ Chou et al ${ }^{11}$ concluded that 3D US may be a useful adjunctive tool in refining 2D ultrasonographic techniques to identify the extent and degree of placental invasion of the bladder. A recent publication by Shih and colleagues ${ }^{15}$ assessed the role of 3D power Doppler in the antenatal diagnosis of placenta accreta and compared its diagnostic performance with grayscale and color Doppler US. The final results revealed that placenta accreta and its variants (including increta and percreta) were confirmed in 39 patients at the time of cesarean delivery. Based on receiver-operating characteristics analysis, "numerous coherent vessels" visualized using 3D power Doppler in the basal view was the best single criterion for the diagnosis of placenta accreta, with a sensitivity of $97 \%$ and a specificity of $92 \%$. If Shih et $\mathrm{al}^{15}$ considered the presence of at least one criterion to be diagnostic when using each ultrasound technique, then 3D power Doppler would have the best positive predictive value (76\%), followed by grayscale (51\%) and color Doppler (47\%). Chou et al ${ }^{14}$ recommended that timely pregnancy termination is an appropriate management option because of decreased morbidity and mortality. In this issue, two patients received total hysterectomy and the other five received cesarean hysterectomy among those seven patients.

Our patient had a cesarean hysterectomy with significant blood loss and had received massive blood transfusion intraoperatively. The urologist were available during the operation and proper bladder dissection was performed with identification of both ureters. It was a good practice to involve the urologist, the vascular surgeon, senior anesthetist, the interventional radiologist and hematologist for the management of this patient as with multidisciplinary approach.
Timely pregnancy termination is an appropriate management option to decrease morbidity and mortality. Indeed, correct antenatal diagnosis of the extent of placental invasion before delivery allowed us to be best prepared. We recommend making rapid transfusion equipment readily available because of the speed with which massive hemorrhage can occur at the time of wide dissection of the vascular lower uterine segment and parametrial areas from the tightly adherent bladder flap. Although the meticulous techniques of cesarean hysterectomy as described by Price et $a l,{ }^{16}$ Catanzarite et $\mathrm{al}^{17}$ and Pelosi ${ }^{18}$ were followed by highly experienced surgeons in this study, the mean operative blood loss was still massive. Levine et $\mathrm{al}^{19}$ and Zacharias et $\mathrm{al}^{20}$ reported their experience with the use of prophylactic internal iliac artery balloon occlusion catheters in patients equiring a cesarean hysterectomy for placenta accreta. Their preliminary findings suggested that transarterial balloon occlusion procedures did not improve the surgical outcomes. They speculated that this lack of success may reflect their inability to preoperatively select the optimal placement site of the occlusion balloons. Additionally, the rich collateral network of vessels in the pelvis may interfere with the efficacy of selective arterial occlusion. ${ }^{11,19}$

\section{CONCLUSION}

Grayscale, color Doppler and 3D power Doppler US remain the standard screening tool for the diagnosis of invasive placentation. 3D power Doppler would have the best positive predictive value (76\%), followed by grayscale (51\%) and color Doppler (47\%). ${ }^{15}$ In some cases, magnetic resonance imaging may be a useful adjunct to ultrasound in diagnosing invasive placentation prenatally. ${ }^{21}$

3D US can further refine conventional ultrasonographic techniques to reliably assess the presence or absence of placental invasion of the bladder.

The advantage is due to the multiplanar imaging capability of 3D US combined with a dynamic assessment of the uterine wall-bladder interface spatial vascular network. Recognizable 3D photographic images are provided, giving a clear view of the severity of advanced invasive placentation to the patients. Hence, 3D US offers affected patients an improved visual perspective of their pathologic condition compared with 2D US.

The obstetrician is thus better equipped to counsel patients and their families about the risks of potential lifethreatening hemorrhage and massive blood transfusions and to psychologically prepare them for the appropriate surgical interventions. 


\section{REFERENCES}

1. Gorodeski IG, Bahari CM. The effect of placenta previa localization upon maternal and fetal-neonatal outcome. J Perinat Med 1987;15:169-77.

2. Taipale P, Hiilesmaa V, Ylostalo P. Diagnosis of placenta previa by transvaginal sonographic screening at 12-16 weeks in a nonselected population. Obstet Gynecol 1997;89:364-67.

3. Lachman E, Mali A, Gino F, Burstein M, Strak M. Placenta accreta with placenta previa after previous cesarean sections: A growing danger in modern obstetrics. Harefuah 2000;138: 628-31.

4. Chou MM, Ho ESC, Lee YH. Prenatal diagnosis of placenta previa accreta by transabdominal color Doppler ultrasound. Ultrasound Obstet Gynecol 2000;15:28-35.

5. Kirkinen P, Helin-Martikainen HL, Vanninen R, Partanen K. Placenta accreta: Imaging by grayscale and contrast-enhanced color Doppler sonography and magnetic resonance imaging. $\mathrm{J}$ Clin Ultrasound 1998;26:90-94.

6. Sumigama S, Itakura A, Ota T, et al. Placenta previa increta/ percreta in Japan: A retrospective study of ultrasound findings, management and clinical course. J Obstet Gynaecol Res 2007; 33:606-11.

7. Hudon L, Belfort MA, Broome DR. Diagnosis and management of placenta percreta: A review. Obstet Gynecol Surv 1998; 53:509-17.

8. Megier P, Harmas A, Mesnard L, Esperandieu OL, Desroches A. Antenatal diagnosis of placenta percreta using grayscale ultrasonography, color and pulsed Doppler imaging. Ultrasound Obstet Gynecol 2000;15:268.

9. Levine D, Hulka CA, Ludmir J, Li W, Edelman RR. Placenta accreta: Evaluation with color Doppler US, power Doppler US, and MR imaging. Radiology 1997;205:773-76.

10. Te-Yao Hsu. Abnormal invasive placentation: Placenta previa increta and percreta. Taiwan J Obstet Gynecol 2009;48(1).

11. Chou MM, Chen WC, Tseng JJ, Chen YF, Yeh TT, Ho ES. Prenatal detection of bladder wall involvement in invasive placentation with sequential two-dimensional and adjunctive three-dimensional ultrasonography. Taiwan J Obstet Gynecol 2009;48:38-45.

12. Baughman WC, Corteville JE, Shah RR. Placenta Accreta: Spectrum of US and MR Imaging Findings. Radiographics 2008;28(7).

13. Tourne G, Collet F, Seffert P, Veyret C. Place of embolization of the uterine arteries in the management of postpartum haemorrhage: A study of 12 cases. Eur J Obstet Gynecol Reprod Biol 2003;110:29-34.

14. Chou MM, Tseng JJ, Ho ES, Hwang JI. Three-dimensional color power Doppler imaging in the assessment of uteroplacental neovascularization in placenta previa increta/percreta. Am J Obstet Gynecol 2001;185:1257-60.

15. Shih JC, Palacios Jaraquemada JM, Su YN, Shyu MK, Lin CH, Lin SY, Lee CN. Role of three-dimensional power Doppler in the antenatal diagnosis of placenta accreta: Comparison with grayscale and color Doppler techniques. Ultrasound Obstet Gynecol 2009;33:193-203.

16. Price FV, Resnik E, Heller KA, Christopherson WA. Placenta previa percreta involving the urinary bladder: A report of two cases and review of the literature. Obstet Gynecol 1991;78: 508-11.

17. Catanzarite VA, Stanco LM. Schrimmer DR, Conroy C. Managing placenta previa/accreta. Contemp Ob Gyn 1996; 41:66-95.

18. Pelosi MA (3rd), Pelosi MA. Modified cesarean hysterectomy for placenta previa percreta with bladder invasion: Retrovesical lower uterine segment bypass. Obstet Gynecol 1999;93:830-3.

19. Levine AB, Kuhlman K, Bonn J. Placenta accreta: Comparison of cases managed with and without pelvic artery balloon catheters. J Matern Fetal Med 1999;8:173-76.

20. Zacharias N, Gei A, Suarez V, et al. Balloon-tip catheter occlusion of the hypogastric arteries for the management of placenta accreta. Am J Obstet Gynecol 2003;189 (Suppl 1):S128.

21. Lax A, Prince MR, Mennitt KW, Schwebach JR, Budorick NE. The value of specific MRI features in the evaluation of suspected placental invasion. Magn Reson Imaging 2007;25:87-93. 